\title{
Stability of a solution set for parametric generalized vector mixed quasivariational inequality problem
}

Nguyen Van Hung*

*Correspondence:

ngvhungdhdt@yahoo.com Department of Mathematics, Dong Thap University, 783 Pham Huu Lau Street, Ward 6, Cao Lanh, Vietnam

\begin{abstract}
In this paper, we study a class of parametric generalized vector mixed quasivariational inequality problems (in short, (MQVIP)) in Hausdorff topological vector spaces. The upper semicontinuity, closedness, the outer-continuity and the outer-openness of the solution set are obtained. Moreover, a key assumption is introduced by virtue of a parametric gap function. Then, by using the key assumption, we establish that the condition $\left(H_{h}\left(\gamma_{0}, \mu_{0}\right)\right)$ is a sufficient and necessary condition for the lower semicontinuity, the Hausdorff lower semicontinuity, the continuity and Hausdorff continuity of solutions for (MQVIP). The results presented in this paper are new and extend some main results in the literature.
\end{abstract}

MSC: $90 \mathrm{C} 31 ; 49 J 53 ; 49 J 40 ; 49 J 45$

Keywords: parametric generalized vector mixed quasivariational inequality problem; parametric gap function; upper semicontinuity; outer-continuity; outer-openness; lower semicontinuity; Hausdorff lower semicontinuity; continuity; H-continuity

\section{Introduction}

Let $X, Y$ be two Hausdorff topological vector spaces and let $\Lambda, M$ be two topological vector spaces. Let $L(X, Y)$ be the space of all linear continuous operators from $X$ to $Y$. Let $K: X \times \Lambda \rightarrow 2^{X}, T: X \times M \rightarrow 2^{L(X, Y)}$ be set-valued mappings and let $C: X \rightarrow 2^{Y}$ be a set-valued mapping such that $C(x)$ is a closed pointed convex cone with int $C(x) \neq \emptyset$. Let $\Omega: X \times X \times \Lambda \rightarrow X, \Theta: X \times X \times \Lambda \rightarrow Y$ be two continuous vector-valued functions satisfying $\Omega(y, y, \gamma)=0$ and $\Theta(y, y, \gamma)=0$ for each $y \in X, \gamma \in \Lambda$. And let $Q: L(X, Y) \rightarrow$ $L(X, Y), \psi: X \rightarrow X$ be continuous single-valued mappings. Denoting by $\langle z, x\rangle$ the value of a linear operator $z \in L(X ; Y)$ at $x \in X$, we always assume that $\langle\cdot, \cdot\rangle$ is continuous.

For $\gamma \in \Lambda, \mu \in M$, we consider the following parametric generalized vector mixed quasivariational inequality problem (in short, (MQVIP)).

(MQVIP) Find $\bar{x} \in K(\bar{x}, \gamma)$ and $\bar{z} \in T(\bar{x}, \mu)$ such that

$$
\langle Q(\bar{z}), \Omega(y, \psi(\bar{x}), \gamma)\rangle+\Theta(y, \psi(\bar{x}), \gamma) \notin-\operatorname{int} C(\bar{x}), \quad \forall y \in K(\bar{x}, \gamma) .
$$

For each $\gamma \in \Lambda, \mu \in M$, we let $E(\gamma):=\{x \in X \mid x \in K(x, \gamma)\}$ and $\Psi: \Lambda \times M \rightarrow 2^{X}$ be a setvalued mapping such that $\Psi(\gamma, \mu)$ is the solution set of (MQVIP). Throughout this paper, we always assume that $\Psi(\gamma, \mu) \neq \emptyset$ for each $(\gamma, \mu)$ in the neighborhood $\left(\gamma_{0}, \mu_{0}\right) \in \Lambda \times M$.

\section{Springer}

(c) 2013 Hung; licensee Springer. This is an Open Access article distributed under the terms of the Creative Commons Attribution License (http://creativecommons.org/licenses/by/2.0), which permits unrestricted use, distribution, and reproduction in any medium, provided the original work is properly cited. 
Special cases of the problem (MQVIP) are as follows:

(a) If we let $K(x, \gamma)=K(x), \Omega(y, \psi(x), \gamma)=\Omega(y, \psi(x)), \Theta(y, \psi(x), \gamma)=\Theta(\psi(x), y)$, then the problem (MQVIP) is reduced to the following generalized vector mixed general quasi-variational-like inequality problem:

Find $\bar{x} \in X$ such that $\bar{x} \in K(\bar{x})$ and for each $y \in K(\bar{x})$, there exists $\bar{z} \in T(\bar{x})$ satisfying

$$
\langle Q(\bar{z}), \Omega(y, \psi(\bar{x}))\rangle+\Theta(\psi(\bar{x}), y) \notin-\operatorname{int} C(\bar{x})
$$

This problem was studied in [1].

(b) If $Q, \psi$ are identity mappings and $\Omega(y, \psi(x), \gamma)=\Omega(y, x), \Theta(y, \psi(x), \gamma)=\Theta(y, x)$, $K(x, \gamma)=K(\gamma)$, then the problem (MQVIP) is reduced to the following parametric generalized vector quasi-variational-like inequality problem (in short, (PGVQVLIP)):

(PGVQVLIP) Find $\bar{x} \in K(\gamma)$ and $\bar{z} \in T(\bar{x}, \mu)$ such that

$$
\langle\bar{z}, \eta(y, \bar{x})\rangle+\psi(y, \bar{x}) \notin-\operatorname{int} C(\bar{x}), \quad \forall y \in K(\gamma) .
$$

This problem was studied in [2].

(c) If $Q, \psi$ are identity mappings and $K(x, \gamma)=X, T(\bar{x}, \mu)=T(\bar{x})$,

$\Omega(y, \psi(x), \gamma)=\Omega(y, x), \Theta(y, \psi(x), \gamma)=\Theta(y, x)$ and $C(x)=C$ with $C \subseteq Y$ is a pointed, closed and convex cone in $Y$ with int $C \neq \emptyset$, then the problem (MQVIP) is reduced to the following generalized vector variational inequality problem:

Find $\bar{x} \in X$ and $\bar{z} \in T(\bar{x})$ such that

$$
\langle\bar{z}, \eta(y, \bar{x})\rangle+\psi(y, \bar{x}) \notin-\operatorname{int} C, \quad \forall y \in K(\gamma) .
$$

This problem was studied in [3].

(d) If $Q, \psi$ are identity mappings and $\Omega(y, \eta(x), \gamma)=y-x, \Theta(y, \psi(x), \gamma)=0, \Lambda=M$, then the problem (MQVIP) is reduced to the following generalized vector quasivariational inequality problem (in short, (PGVQVI)):

(PGVQVI) Find $\bar{x} \in K(\bar{x}, \gamma)$ and $\bar{z} \in T(\bar{x}, \gamma)$ such that

$$
\langle\bar{z}, y-\bar{x}\rangle \in Y \backslash-\operatorname{int} C(\bar{x}), \quad \forall y \in K(\bar{x}, \gamma) .
$$

This problem was studied in [4].

(e) If $Q, \psi$ are identity mappings and $\Omega(y, \eta(x), \gamma)=y-x, \Theta(y, \psi(x), \gamma)=0$, $K(x, \gamma)=K(\gamma), \Lambda=M$ and $C(x)=C$ with $C \subseteq Y$ is a pointed closed and convex cone in $Y$ with int $C \neq \emptyset$, then the problem (MQVIP) is reduced to the following parametric set-valued weak vector variational inequality (in short, (PSWVVI)): (PSWVVI) Find $\bar{x} \in K(\gamma)$ and $\bar{z} \in T(\bar{x}, \gamma)$ such that

$$
\langle\bar{z}, y-\bar{x}\rangle \notin-\operatorname{int} C, \quad \forall y \in K(\gamma) .
$$

This problem was studied in [5].

(f) If $Q, \psi$ are identity mappings and $\Omega(y, \eta(x), \gamma)=0, \Theta(y, \psi(x), \gamma)=\Theta(x, y, z), \Lambda=M$, then the problem (MQVIP) is reduced to the following parametric generalized vector quasiequilibrium problem (in short, (PGVQEP)): 
(PGVQEP) Find $\bar{x} \in K(\bar{x}, \gamma)$ and $\bar{z} \in T(\bar{x}, \gamma)$ such that

$$
\Theta(x, y, z) \in Y \backslash-\operatorname{int} C(\bar{x}), \quad \forall y \in K(\bar{x}, \gamma) .
$$

This problem was studied in [6].

(g) If $Q, \psi$ are identity mappings and $Y=\mathbb{R}^{n}, C(x)=\mathbb{R}_{+}^{n}, \Lambda=M, K(x, \gamma)=K(\gamma)$, $\Omega(y, \eta(x), \gamma)=y-x, \Theta(y, \psi(x), \gamma)=0$ and $T: X \times \Lambda \rightarrow L\left(X, \mathbb{R}^{n}\right)$, then the problem (MQVIP) is reduced to the parametric weak vector variational inequality problem (in short, (PWVVI)):

(PWVVI) Find $\bar{x} \in K(\gamma)$ such that

$$
\langle T(\bar{x}, \gamma), y-x\rangle \notin-\operatorname{int} \mathbb{R}_{+}^{n}, \quad \forall y \in K(\gamma) .
$$

This problem was studied in [7].

Stability of solutions for the parametric generalized vector mixed quasivariational inequality problem is an important topic in optimization theory and applications. Recently, the continuity, especially the upper semicontinuity, the lower semicontinuity and the Hausdorff lower semicontinuity of the solution sets for parametric optimization problems, parametric vector variational inequality problems and parametric vector quasiequilibrium problems have been studied in the literature; see [2, 4-17] and the references therein.

The structure of our paper is as follows. In the first part of this article, we introduce the model parametric generalized vector mixed quasivariational inequality problems. In Section 2, we recall definitions for later uses. In Section 3, we establish the upper semicontinuity, closedness, the outer-continuity and the outer-openness, and in Section 4, we establish that the condition $\left(H_{h}\left(\gamma_{0}, \mu_{0}\right)\right)$ is a sufficient and necessary condition for the lower semicontinuity, the Hausdorff lower semicontinuity, the continuity and Hausdorff continuity of the solution set for the parametric generalized vector mixed quasivariational inequality problem in Hausdorff topological vector spaces.

\section{Preliminaries}

In this section, we recall some basic definitions and some of their properties.

First, we recall two limits in $[18,19]$. Let $X$ and $Y$ be two topological vector spaces and $G: X \rightarrow 2^{Y}$ be a multifunction. The superior limit and the superior open limit of $G$ are defined as

$$
\begin{aligned}
\limsup _{x \rightarrow x_{0}} G(x):= & \left\{y \in Y \mid \exists x_{v} \rightarrow x_{0}, \exists y_{v} \in G\left(x_{v}\right): y_{v} \rightarrow y, \forall v\right\}, \\
\operatorname{limsupo}_{x \rightarrow x_{0}} G(x):= & \{y \in Y \mid \text { there are an open neighborhood } U \text { of } y \text { and a net } \\
& \left.\left\{x_{v}\right\} \subseteq X, x_{v} \neq x_{0} \text { converging to } x_{0} \text { such that } U \subseteq G\left(x_{v}\right), \forall v\right\} .
\end{aligned}
$$

Definition $2.1([18,20,21])$ Let $X$ and $Y$ be topological vector spaces and $G: X \rightarrow 2^{Y}$ be a multifunction.

(i) $G$ is said to be outer-continuous at $x_{0} \in X$ if $\limsup _{x \rightarrow x_{0}} G(x) \subseteq G\left(x_{0}\right)$. $G$ is said to be outer-continuous in $X$ if it is outer-continuous at each $x_{0} \in X$.

(ii) $G$ is said to be outer-open at $x_{0} \in X$ if $\operatorname{limsupo}_{x \rightarrow x_{0}} G(x) \subseteq G\left(x_{0}\right)$. $G$ is said to be outer-open in $X$ if it is outer-open at each $x_{0} \in X$. 
(iii) $G$ is said to be lower semicontinuous (lsc) at $x_{0} \in X$ if $G\left(x_{0}\right) \cap U \neq \emptyset$ for some open set $U \subseteq Y$ implies the existence of a neighborhood $N$ of $x_{0}$ such that $G(x) \cap U \neq \emptyset$, $\forall x \in N$. $G$ is said to be lower semicontinuous in $X$ if it is lower semicontinuous at each $x_{0} \in X$.

(iv) $G$ is said to be upper semicontinuous (usc) at $x_{0} \in X$ if for each open set $U \supseteq G\left(x_{0}\right)$, there is a neighborhood $N$ of $x_{0}$ such that $U \supseteq G(x), \forall x \in N$. $G$ is said to be upper semicontinuous in $X$ if it is upper semicontinuous at each $x_{0} \in X$.

(v) $G$ is said to be Hausdorff upper semicontinuous (H-usc) at $x_{0} \in X$ if for each neighborhood $B$ of the origin in $Z$, there exists a neighborhood $N$ of $x_{0}$ such that $G(x) \subseteq G\left(x_{0}\right)+B, \forall x \in N$. $G$ is said to be Hausdorff upper semicontinuous in $X$ if it is Hausdorff upper semicontinuous at each $x_{0} \in X$.

(vi) $G$ is said to be Hausdorfflower semicontinuous (H-lsc) at $x_{0} \in X$ if for each neighborhood $B$ of the origin in $Y$, there exists a neighborhood $N$ of $x_{0}$ such that $G\left(x_{0}\right) \subseteq G(x)+B, \forall x \in N$. $G$ is said to be Hausdorff lower semicontinuous in $X$ if it is Hausdorff lower semicontinuous at each $x_{0} \in X$.

(vii) $G$ is said to be continuous at $x_{0} \in X$ if it is both lsc and usc at $x_{0}$ and to be $H$-continuous at $x_{0} \in X$ if it is both H-lsc and H-usc at $x_{0} . G$ is said to be continuous in $X$ if it is both lsc and usc at each $x_{0} \in X$ and to be $\mathrm{H}$-continuous in $X$ if it is both $\mathrm{H}$-lsc and $\mathrm{H}$-usc at each $x_{0} \in X$.

(viii) $G$ is said to be closed at $x_{0} \in X$ if and only if $\forall x_{n} \rightarrow x_{0}, \forall y_{n} \rightarrow y_{0}$ such that $y_{n} \in G\left(x_{n}\right)$, we have $y_{0} \in G\left(x_{0}\right)$. G is said to be closed in $X$ if it is closed at each $x_{0} \in X$.

Lemma $2.2([20,21])$ Let $X$ and $Y$ be topological vector spaces and $G: X \rightarrow 2^{Y}$ be a multifunction.

(i) If $G$ is usc at $x_{0}$, then $G$ is H-usc at $x_{0}$. Conversely if $G$ is H-usc at $x_{0}$ and if $G\left(x_{0}\right)$ is compact, then $G$ is usc at $x_{0}$;

(ii) If $G$ is $H$-lsc at $x_{0}$ then $G$ is lsc at $x_{0}$. The converse is true if $G\left(x_{0}\right)$ is compact;

(iii) If $Y$ is compact and $G$ is closed at $x_{0}$, then $G$ is usc at $x_{0}$;

(iv) If $G$ is usc at $x_{0}$ and $G\left(x_{0}\right)$ is closed, then $G$ is closed at $x_{0}$;

(v) If $G$ has compact values, then $G$ is usc at $x_{0}$ if and only if, for each net $\left\{x_{\alpha}\right\} \subseteq X$ which converges to $x_{0}$ and for each net $\left\{y_{\alpha}\right\} \subseteq G\left(x_{\alpha}\right)$, there are $y \in G\left(x_{0}\right)$ and $a$ subnet $\left\{y_{\beta}\right\}$ of $\left\{y_{\alpha}\right\}$ such that $y_{\beta} \rightarrow y$.

Lemma $2.3([22,23])$ Let $e: X \rightarrow Y$ be a vector-valued mapping and for any $x \in X, e \in$ int $C(x)$. The nonlinear scalarization function $\xi_{e}: X \times Y \rightarrow \mathbb{R}$ defined by $\xi_{e}(x, y):=\inf \{r \in$ $\mathbb{R}: y \in \operatorname{re}(x)-C(x)\}$ has the following properties:

(i) $\xi_{e}(x, y)<r \Leftrightarrow y \in r e-\operatorname{int} C(x)$;

(ii) $\xi_{e}(x, y) \geq r \Leftrightarrow y \notin r e-\operatorname{int} C(x)$.

Lemma 2.4 (See $[4,23])$ Let $X$ and $Y$ be two locally convex Hausdorff topological vector spaces, and let $C: X \rightarrow 2^{Y}$ be a set-valued mapping such that, for each $x \in X, C(x)$ is a proper, closed, convex cone in $Y$ with int $C(x) \neq \emptyset$. Furthermore, let $e: X \rightarrow Y$ be the continuous selection of the set-valued map int $C(\cdot)$. Define a set-valued mapping $V: X \rightarrow 2^{Y}$ by $V(x)=Y \backslash \operatorname{int} C(x)$ for $x \in X$. We have

(i) If $V(\cdot)$ is usc in $X$, then $\xi_{e}(\cdot, \cdot)$ is upper semicontinuous in $X \times Y$;

(ii) If $C(\cdot)$ is usc in $X$, then $\xi_{e}(\cdot, \cdot)$ is lower semicontinuous in $X \times Y$. 
From Lemma 2.4, we know that if $V(\cdot)$ and $C(\cdot)$ are both usc in $X$, then $\xi_{e}(\cdot, \cdot)$ is continuous in $X \times Y$.

Now we suppose that $K(x, \gamma)$ and $T(x, \mu)$ are compact sets for any $(x, \gamma) \in X \times \Lambda$ and $(x, \mu) \in X \times M$. We define a function $h: X \times \Lambda \times M \rightarrow \mathbb{R}$ as follows:

$$
h(x, \gamma, \mu)=\min _{z \in T(x, \mu)} \max _{y \in K(x, \gamma)}\left\{-\xi_{e}(x,\langle Q(z), \Omega(y, \psi(x), \gamma)\rangle+\Theta(y, \psi(x), \gamma))\right\} .
$$

Since $K(x, \gamma)$ and $T(x, \mu)$ are compact sets, $h(x, \gamma, \mu)$ is well defined.

\section{Lemma 2.5}

(i) $h(x, \gamma, \mu) \geq 0$ for all $x \in E(\gamma)$;

(ii) $h\left(x_{0}, \gamma_{0}, \mu_{0}\right)=0$ if and only if $x_{0} \in \Psi\left(\gamma_{0}, \mu_{0}\right)$.

Proof We define a function $h_{1}: X \times L(X, Y) \rightarrow \mathbb{R}$ as follows:

$$
h_{1}(x, z)=\max _{y \in K(x, \gamma)}\left\{-\xi_{e}(x,\langle Q(z), \Omega(y, \psi(x), \gamma)\rangle+\Theta(y, \psi(x), \gamma))\right\} .
$$

(i) It is easy to see that $h_{1}(x, z) \geq 0$. Suppose to the contrary that there exists $x_{0} \in E(\gamma)$ and $z_{0} \in T\left(x_{0}, \mu\right)$ such that $h_{1}\left(x_{0}, z_{0}\right)<0$, then

$$
\begin{aligned}
0 & >h_{1}\left(x_{0}, z_{0}\right)=\max _{y \in K\left(x_{0}, \gamma\right)}\left\{-\xi_{e}\left(x_{0},\left\langle Q\left(z_{0}\right), \Omega\left(y, \psi\left(x_{0}\right), \gamma\right)\right\rangle+\Theta\left(y, \psi\left(x_{0}\right), \gamma\right)\right)\right\} \\
& \geq-\xi_{e}\left(x_{0},\left\langle Q\left(z_{0}\right), \Omega\left(y, \psi\left(x_{0}\right), \gamma\right)\right\rangle+\Theta\left(y, \psi\left(x_{0}\right), \gamma\right)\right) .
\end{aligned}
$$

When $\psi\left(x_{0}\right)=y$, we have

$$
\begin{aligned}
\xi_{e} & \left(x_{0},\left\langle Q\left(z_{0}\right), \Omega(y, y, \gamma)\right\rangle+\Theta(y, y, \gamma)\right) \\
& =\xi_{e}\left(x_{0}, 0\right) \\
& =\inf \left\{r \in \mathbb{R}: 0 \in \operatorname{re}\left(x_{0}\right)-C\left(x_{0}\right)\right\} \\
& =\inf \left\{r \in \mathbb{R}:-r e\left(x_{0}\right) \in-C\left(x_{0}\right)\right\} \\
& =\inf \{r \in \mathbb{R}: r \geq 0\}=0,
\end{aligned}
$$

which is a contradiction. Hence,

$$
\begin{aligned}
h_{1}(x, z)= & \max _{y \in K(x, \gamma)}\left\{-\xi_{e}(x,\langle Q(z), \Omega(y, \psi(x), \gamma)\rangle+\Theta(y, \psi(x), \gamma))\right\} \geq 0, \\
& x \in E(\gamma), z \in T(x, \gamma) .
\end{aligned}
$$

Thus, since $z \in T(x, \mu)$ is arbitrary, we have

$$
h(x, \gamma, \mu)=\min _{z \in T(x, \mu)} \max _{y \in K(x, \gamma)}\left\{-\xi_{e}(x,\langle Q(z), \Omega(y, \psi(x), \gamma)\rangle+\Theta(y, \psi(x), \gamma))\right\} \geq 0 .
$$

(ii) By definition, $h\left(x_{0}, \gamma_{0}, \mu_{0}\right)=0$ if and only if there exists $z_{0} \in T\left(x_{0}, \mu_{0}\right)$ such that $h_{1}\left(x_{0}, z_{0}\right)=0$, i.e.,

$$
\max _{y \in K\left(x_{0}, \gamma_{0}\right)}\left\{-\xi_{e}\left(x_{0},\left\{Q\left(z_{0}\right), \Omega\left(y, \psi\left(x_{0}\right), \gamma_{0}\right)\right)+\Theta\left(y, \psi\left(x_{0}\right), \gamma_{0}\right)\right)\right\}=0, \quad x_{0} \in E\left(\gamma_{0}\right)
$$


if and only if, for any $y \in K\left(x_{0}, \gamma_{0}\right)$,

$$
-\xi_{e}\left(x_{0},\left\langle Q\left(z_{0}\right), \Omega\left(y, \psi\left(x_{0}\right), \gamma_{0}\right)\right\rangle+\Theta\left(y, \psi\left(x_{0}\right), \gamma_{0}\right)\right) \leq 0,
$$

or

$$
\xi_{e}\left(x_{0},\left\langle Q\left(z_{0}\right), \Omega\left(y, \psi\left(x_{0}\right), \gamma_{0}\right)\right\rangle+\Theta\left(y, \psi\left(x_{0}\right), \gamma_{0}\right)\right) \geq 0 \text {. }
$$

By Lemma 2.3(ii), if and only if

$$
\left.\left\langle Q\left(z_{0}\right), \Omega\left(y, \psi\left(x_{0}\right), \gamma_{0}\right)\right\rangle+\Theta\left(y, \psi\left(x_{0}\right), \gamma_{0}\right)\right) \notin-\operatorname{int} C\left(x_{0}\right), \quad \forall y \in K\left(x_{0}, \gamma_{0}\right),
$$

that is, $x_{0} \in \Psi\left(\gamma_{0}, \mu_{0}\right)$.

We may call the function $h(\cdot, \cdot, \cdot)$ a parametric gap function for (MQVIP) if the properties of Lemma 2.5 are satisfied. Many authors have studied the gap functions for vector equilibrium problems and vector variational inequalities; see [3, 24-27] and the references therein.

Example 2.6 Let $\psi, Q$ be identity mappings and $X=Y=\mathbb{R}, \Lambda=M=[0,1], C(x)=\mathbb{R}_{+}$, $K(x, \gamma)=[0,1], T(x, \gamma)=\left\{2^{\gamma^{2}+2}\right\}, \Omega(y, \psi(x), \gamma)=\Theta(y, \psi(x), \gamma)=y-x$. Now we consider the problem (MQVIP) of finding $x \in K(x, \gamma)$ and $z \in T(x, \gamma)$ such that

$$
\langle Q(z), \Omega(y, \psi(x), \gamma)\rangle+\Theta(y, \psi(x), \gamma)=2^{\gamma^{2}+2}(y-x)+y-x \notin-\operatorname{int} \mathbb{R}_{+} .
$$

It follows from a direct computation $\Psi(\gamma, \mu)=\{0\}$ for all $\gamma \in[0,1]$. Now we show that $h(\cdot, \cdot, \cdot)$ is a parametric gap function of (MQVIP). Indeed, taking $e=1 \in \operatorname{int} \mathbb{R}_{+}$, we have

$$
\begin{aligned}
h(x, \gamma, \mu) & =\min _{z \in T(x, \mu)} \max _{y \in K(x, \gamma)}\left\{-\xi_{e}(x|Q(z), \Omega(y, \psi(x), \gamma)\rangle+\Theta(y, \psi(x), \gamma))\right\} \\
& =\max _{y \in K(x, \gamma)}\left(\left(1+2^{\gamma^{2}+2}\right)(x-y)\right) \\
& = \begin{cases}0 & \text { if } x=0, \\
\left(1+2^{\gamma^{2}+2}\right) x & \text { if } x \in(0,1] .\end{cases}
\end{aligned}
$$

Hence, $h(\cdot, \cdot, \cdot)$ is a parametric gap function of (MQVIP).

The following lemma gives a sufficient condition for the parametric gap function $h(\cdot, \cdot, \cdot)$ is continuous in $X \times \Lambda \times M$.

Lemma 2.7 Consider (MQVIP). If the following conditions hold:

(i) $K(\cdot, \cdot)$ is continuous with compact values in $\Lambda$;

(ii) $T(\cdot, \cdot)$ is continuous with compact values in $X \times \Lambda$;

(iii) $C(\cdot)$ is upper semicontinuous in $X$ and $e(\cdot) \in \operatorname{int} C(\cdot)$ is continuous in $X$.

Then $h(\cdot, \cdot, \cdot)$ is continuous in $X \times \Lambda \times M$. 
Proof First we prove that $h(\cdot, \cdot, \cdot)$ is lower semicontinuous in $X \times \Lambda \times M$. Indeed, we let $r \in \mathbb{R}$. Suppose that $\left\{\left(x_{\alpha}, \gamma_{\alpha}, \mu_{\alpha}\right)\right\} \subseteq X \times \Lambda \times M$ satisfies

$$
h\left(x_{\alpha}, \gamma_{\alpha}, \mu_{\alpha}\right) \leq r, \quad \forall \alpha
$$

and

$$
\left(x_{\alpha}, \gamma_{\alpha}, \mu_{\alpha}\right) \rightarrow\left(x_{0}, \gamma_{0}, \mu_{0}\right) \quad \text { as } \alpha \rightarrow \infty \text {. }
$$

It follows that

$$
\begin{aligned}
& h\left(x_{\alpha}, \gamma_{\alpha}, \mu_{\alpha}\right) \\
& \quad=\min _{z \in T\left(x_{\alpha}, \mu_{\alpha}\right)} \max _{y \in K\left(x_{\alpha}, \gamma_{\alpha}\right)}\left\{-\xi_{e}\left(x_{\alpha},\left\langle Q(z), \Omega\left(y, \psi\left(x_{\alpha}\right), \gamma_{\alpha}\right)\right\rangle+\Theta\left(y, \psi\left(x_{\alpha}\right), \gamma_{\alpha}\right)\right)\right\} \leq r .
\end{aligned}
$$

We define the function $g: X \times L(X, Y) \times \Lambda \times M \rightarrow \mathbb{R}$ by

$$
g(x, z, \gamma, \mu)=\max _{y \in K(x, \gamma)}\left\{-\xi_{e}(x,\langle Q(z), \Omega(y, \psi(x), \gamma)\rangle+\Theta(y, \psi(x), \gamma))\right\}, \quad x \in E(\gamma) .
$$

By the continuity of $\psi(\cdot), \Omega(\cdot, \cdot, \cdot), \Theta(\cdot, \cdot, \cdot), \xi_{e}(\cdot, \cdot)$ and since $K(\cdot, \cdot)$ is continuous with compact values in $X \times \Lambda$, thus, by Proposition 23 in Section 1 of Chapter 3 [20], we can deduce that $g(x, z, \gamma, \mu)$ is continuous with respect to $(x, z, \gamma, \mu)$. By the compactness of $T\left(x_{\alpha}, \mu_{\alpha}\right)$, there exists $z_{\alpha} \in T\left(x_{\alpha}, \mu_{\alpha}\right)$ such that

$$
\begin{aligned}
h\left(x_{\alpha}, \gamma_{\alpha}, \mu_{\alpha}\right) & =\min _{z \in T\left(x_{\alpha}, \mu_{\alpha}\right)} \max _{y \in K\left(x_{\alpha}, \gamma_{\alpha}\right)}\left\{-\xi_{e}\left(x_{\alpha},\left\langle Q(z), \Omega\left(y, \psi\left(x_{\alpha}\right), \gamma_{\alpha}\right)\right\rangle+\Theta\left(y, \psi\left(x_{\alpha}\right), \gamma_{\alpha}\right)\right)\right\} \\
& =g\left(x_{\alpha}, z_{\alpha}, \gamma_{\alpha}, \mu_{\alpha}\right) \\
& =\max _{y \in K\left(x_{\alpha}, \gamma_{\alpha}\right)}\left\{-\xi_{e}\left(x_{\alpha},\left\{Q\left(z_{\alpha}\right), \Omega\left(y, \psi\left(x_{\alpha}\right), \gamma_{\alpha}\right)\right\rangle+\Theta\left(y, \psi\left(x_{\alpha}\right), \gamma_{\alpha}\right)\right)\right\} \leq r .
\end{aligned}
$$

Since $K(\cdot, \cdot)$ is lower semicontinuous in $X \times \Lambda$, for any $y_{0} \in K\left(x_{0}, \gamma_{0}\right)$, there exists $y_{\alpha} \in$ $K\left(x_{\alpha}, \gamma_{\alpha}\right)$ such that $y_{\alpha} \rightarrow y_{0}$. For $y_{\alpha} \in K\left(x_{\alpha}, \gamma_{\alpha}\right)$, we have

$$
-\xi_{e}\left(x_{\alpha},\left\langle Q\left(z_{\alpha}\right), \Omega\left(y_{\alpha}, \psi\left(x_{\alpha}\right), \gamma_{\alpha}\right)\right\rangle+\Theta\left(y_{\alpha}, \psi\left(x_{\alpha}\right), \gamma_{\alpha}\right)\right) \leq r
$$

Since $T(\cdot, \cdot)$ is upper semicontinuous with compact values in $X \times M$, there exists $z_{0} \in$ $T\left(x_{0}, \mu_{0}\right)$ such that $z_{\alpha} \rightarrow z_{0}$ (taking a subnet $\left\{z_{\beta}\right\}$ of $\left\{z_{\alpha}\right\}$ if necessary) as $\alpha \rightarrow \infty$. From the continuity of $\xi_{e}(\cdot,(Q(\cdot, \psi(\cdot), \cdot)+\Theta(\cdot, \psi(\cdot), \cdot)))$, taking the limit in $(2.1)$, we have

$$
-\xi_{e}\left(x_{0},\left\langle Q\left(z_{0}\right), \Omega\left(y_{0}, \psi\left(x_{0}\right), \gamma_{0}\right)\right\rangle+\Theta\left(y_{0}, \psi\left(x_{0}\right), \gamma_{0}\right)\right) \leq r
$$

Since $y_{0} \in K\left(x_{0}, \gamma_{0}\right)$ is arbitrary, it follows from (2.2) that

$$
g\left(x_{0}, z_{0}, \gamma_{0}, \mu_{0}\right)=\max _{y \in K\left(x_{0}, \gamma_{0}\right)}\left\{-\xi_{e}\left(x_{0},\left\langle Q\left(z_{0}\right), \Omega\left(y, \psi\left(x_{0}\right), \gamma_{0}\right)\right\rangle+\Theta\left(y, \psi\left(x_{0}\right), \gamma_{0}\right)\right)\right\} \leq r .
$$

And so, for any $z_{0} \in T\left(x_{0}, \mu_{0}\right)$, we have

$$
h\left(x_{0}, \gamma_{0}, \mu_{0}\right)=\min _{z \in T\left(x_{0}, \mu_{0}\right)} \max _{y \in K\left(x_{0}, \gamma_{0}\right)}\left\{-\xi_{e}\left(x_{0},\left\langle Q(z), \Omega\left(y, \psi\left(x_{0}\right), \gamma_{0}\right)\right\rangle+\Theta\left(y, \psi\left(x_{0}\right), \gamma_{0}\right)\right)\right\} \leq r .
$$


This proves that, for $r \in \mathbb{R}$, the level set $\{(x, \gamma, \mu) \mid h(x, \gamma, \mu) \leq r\}$ is closed. Hence, $h(\cdot, \cdot, \cdot)$ is lower semicontinuous in $X \times X \times \Lambda$.

Next, we need to prove that $h(\cdot, \cdot, \cdot)$ is upper semicontinuous in $X \times \Lambda \times M$. Indeed, let $r \in \mathbb{R}$. Suppose that $\left\{\left(x_{\alpha}, \gamma_{\alpha}, \mu_{\alpha}\right)\right\} \subseteq X \times \Lambda \times M$ satisfies

$$
h\left(x_{\alpha}, \gamma_{\alpha}, \mu_{\alpha}\right) \geq r, \quad \forall \alpha
$$

and

$$
\left(x_{\alpha}, \gamma_{\alpha}, \mu_{\alpha}\right) \rightarrow\left(x_{0}, \gamma_{0}, \mu_{0}\right) \quad \text { as } \alpha \rightarrow \infty,
$$

then

$$
\min _{z \in T\left(x_{\alpha}, \mu_{\alpha}\right)} \max _{y \in K\left(x_{\alpha}, \gamma_{\alpha}\right)}\left\{-\xi_{e}\left(x_{\alpha},\left\langle Q(z), \Omega\left(y, \psi\left(x_{\alpha}\right), \gamma_{\alpha}\right)\right)+\Theta\left(y, \psi\left(x_{\alpha}\right), \gamma_{\alpha}\right)\right)\right\} \geq r
$$

and so, for any $z \in T\left(x_{\alpha}, \mu_{\alpha}\right)$, we have

$$
\max _{y \in K\left(x_{\alpha}, \gamma_{\alpha}\right)}\left\{-\xi_{e}\left(x_{\alpha},\left\langle Q(z), \Omega\left(y, \psi\left(x_{\alpha}\right), \gamma_{\alpha}\right)\right\rangle+\Theta\left(y, \psi\left(x_{\alpha}\right), \gamma_{\alpha}\right)\right)\right\} \geq r
$$

Since $T(\cdot, \cdot)$ is lower semicontinuous in $X \times M$, for any $z_{0} \in T\left(x_{0}, \mu_{0}\right)$, there exists $z_{\alpha} \in$ $T\left(x_{\alpha}, \mu_{\alpha}\right)$ such that $z_{\alpha} \rightarrow z_{0}$ as $\alpha \rightarrow \infty$. Since $z_{\alpha} \in T\left(x_{\alpha}, \mu_{\alpha}\right)$, it follows (2.3) that

$$
\max _{y \in K\left(x_{\alpha}, \gamma_{\alpha}\right)}\left\{-\xi_{e}\left(x_{\alpha},\left\langle Q\left(z_{\alpha}\right), \Omega\left(y, \psi\left(x_{\alpha}\right), \gamma_{\alpha}\right)\right\rangle+\Theta\left(y, \psi\left(x_{\alpha}\right), \gamma_{\alpha}\right)\right)\right\} \geq r
$$

By the compactness of $K(\cdot, \cdot)$, there exists $y_{\alpha} \in K\left(x_{\alpha}, \gamma_{\alpha}\right)$ such that

$$
-\xi_{e}\left(x_{\alpha},\left\langle Q\left(z_{\alpha}\right), \Omega\left(y_{\alpha}, \psi\left(x_{\alpha}\right), \gamma_{\alpha}\right)\right\rangle+\Theta\left(y_{\alpha}, \psi\left(x_{\alpha}\right), \gamma_{\alpha}\right)\right) \geq r .
$$

Since $K(\cdot, \cdot)$ is upper semicontinuous with compact values, there exists $y_{0} \in K\left(x_{0}, \gamma_{0}\right)$ such that $y_{\alpha} \rightarrow y_{0}$ (taking a subnet $\left\{y_{\beta}\right\}$ of $\left\{y_{\alpha}\right\}$ if necessary) as $\alpha \rightarrow \infty$. From the continuity of $\xi_{e}(\cdot,(Q(\cdot, \psi(\cdot), \cdot)+\Theta(\cdot, \psi(\cdot), \cdot)))$, taking the limit in (2.5), we have

$$
-\xi_{e}\left(x_{0},\left\langle Q\left(z_{0}\right), \Omega\left(y_{0}, \psi\left(x_{0}\right), \gamma_{0}\right)\right\rangle+\Theta\left(y_{0}, \psi\left(x_{0}\right), \gamma_{0}\right)\right) \geq r .
$$

For any $y \in K\left(x_{0}, \gamma_{0}\right)$, we have

$$
\max _{y \in K\left(x_{0}, \gamma_{0}\right)}\left\{-\xi_{e}\left(x_{0},\left\langle Q\left(z_{0}\right), \Omega\left(y, \psi\left(x_{0}\right), \gamma_{0}\right)\right\rangle+\Theta\left(y, \psi\left(x_{0}\right), \gamma_{0}\right)\right)\right\} \geq r .
$$

Since $z \in T\left(x_{0}, \gamma_{0}\right)$ is arbitrary, it follows from (2.6) that

$$
h\left(x_{0}, \gamma_{0}, \mu_{0}\right)=\min _{z \in T\left(x_{0}, \mu_{0}\right)} \max _{y \in K\left(x_{0}, \gamma_{0}\right)}\left\{-\xi_{e}\left(x_{0},\left\langle Q(z), \Omega\left(y, \psi\left(x_{0}\right), \gamma_{0}\right)\right\rangle+\Theta\left(y, \psi\left(x_{0}\right), \gamma_{0}\right)\right)\right\} \geq r .
$$

This proves that, for $r \in \mathbb{R}$, the level set $\{(x, \gamma, \mu) \mid h(x, \gamma, \mu) \geq r\}$ is closed. Hence, $h(\cdot, \cdot, \cdot)$ is upper semicontinuous in $X \times \Lambda \times M$. 
Remark 2.8 In special cases as those in Section 1 (d), (e) and (f),

(i) Lemma 2.5 extends Proposition 4.1 of Chen et al. in [4] and Lemma 2.6 of Zhong-Huang in [6].

(ii) Lemma 2.7 extends Lemma 4.2 of Chen et al. in [4], Lemma 2.7 of Zhong-Huang in [5] and Lemma 2.8 of Zhong-Huang in [6].

\section{Upper semicontinuity of a solution set}

In this section, we establish the upper semicontinuity, closedness, outer-continuity and outer-openess of the solution set for the parametric generalized vector mixed quasivariational inequality problem (MQVIP).

Theorem 3.1 Assume for the problem (MQVIP) that

(i) $E(\cdot)$ is upper semicontinuous with compact values in $\Lambda$ and $K(\cdot, \cdot)$ is lower semicontinuous in $X \times \Lambda$;

(ii) $T(\cdot, \cdot)$ is upper semicontinuous with compact values in $X \times M$;

(iii) $W(\cdot)=Y \backslash-\operatorname{int} C(\cdot)$ is closed in $X$.

Then $\Psi(\cdot, \cdot)$ is upper semicontinuous in $\Lambda \times M$. Moreover, $\Psi\left(\gamma_{0}, \mu_{0}\right)$ is a compact set and $\Psi(\cdot, \cdot)$ is closed in $\Lambda \times M$.

Proof First we prove that $\Psi(\cdot, \cdot)$ is upper semicontinuous in $\Lambda \times M$. Indeed, we suppose that $\Psi(\cdot, \cdot)$ is not upper semicontinuous at $\left(\gamma_{0}, \mu_{0}\right)$, i.e., there is an open subset $V$ of $\Psi\left(\gamma_{0}, \mu_{0}\right)$ such that for all nets $\left\{\left(\gamma_{\alpha}, \mu_{\alpha}\right)\right\}$ convergent to $\left(\gamma_{0}, \mu_{0}\right)$, there is $x_{\alpha} \in \Psi\left(\gamma_{\alpha}, \mu_{\alpha}\right)$, $x_{\alpha} \notin V, \forall \alpha$. By the upper semicontinuity of $E(\cdot)$ in $\Lambda$ and the compactness of $E(\gamma)$, one can assume that $x_{\alpha} \rightarrow x_{0} \in E\left(\gamma_{0}\right)$ (taking a subnet if necessary). Now we show that $x_{0} \in \Psi\left(\gamma_{0}, \mu_{0}\right)$. If $x_{0} \notin \Psi\left(\gamma_{0}, \mu_{0}\right)$, then $\forall z_{0} \in T\left(x_{0}, \mu_{0}\right), \exists y_{0} \in K\left(x_{0}, \gamma_{0}\right)$ such that

$$
\left\langle Q\left(z_{0}\right), \Omega\left(y_{0}, \psi\left(x_{0}\right), \gamma_{0}\right)\right\rangle+\Theta\left(y_{0}, \psi\left(x_{0}\right), \gamma_{0}\right) \in-\operatorname{int} C\left(x_{0}\right) .
$$

By the lower semicontinuity of $K(\cdot, \cdot)$ at $\left(x_{0}, \gamma_{0}\right)$, there exists $y_{\alpha} \in K\left(x_{\alpha}, \gamma_{\alpha}\right)$ such that $y_{\alpha} \rightarrow y_{0}$. Since $x_{\alpha} \in \Psi\left(\gamma_{\alpha}, \mu_{\alpha}\right)$, there exists $z_{\alpha} \in T\left(x_{\alpha}, \mu_{\alpha}\right)$ such that

$$
\left\langle Q\left(z_{\alpha}\right), \Omega\left(y_{\alpha}, \psi\left(x_{\alpha}\right), \gamma_{\alpha}\right)\right\rangle+\Theta\left(y_{\alpha}, \psi\left(x_{\alpha}\right), \gamma_{\alpha}\right) \notin-\operatorname{int} C\left(x_{\alpha}\right) .
$$

Since $T(\cdot, \cdot)$ is upper semicontinuous and with compact values in $X \times M$, one has $z_{0} \in$ $T\left(x_{0}, \mu_{0}\right)$ such that $z_{\alpha} \rightarrow z_{0}$ (can take a subnet if necessary) and since $Q(\cdot), \Omega(\cdot, \psi(\cdot), \cdot)$ are continuous, we have

$$
\left\langle Q\left(z_{\alpha}\right), \Omega\left(y_{\alpha}, \psi\left(x_{\alpha}\right), \gamma_{\alpha}\right)\right\rangle \rightarrow\left\langle Q\left(z_{0}\right), \Omega\left(y_{0}, \psi\left(x_{0}\right), \gamma_{0}\right)\right\rangle .
$$

It follows from the continuity of $\Theta(\cdot, \psi(\cdot), \cdot)$ that

$$
\begin{aligned}
& \left\langle Q\left(z_{\alpha}\right), \Omega\left(y_{\alpha}, \psi\left(x_{\alpha}\right), \gamma_{\alpha}\right)\right\rangle+\Theta\left(y_{\alpha}, \psi\left(x_{\alpha}\right), \gamma_{\alpha}\right) \\
& \quad \rightarrow\left\langle Q\left(z_{0}\right), \Omega\left(y_{0}, \psi\left(x_{0}\right), \gamma_{0}\right)\right\rangle+\Theta\left(y_{0}, \psi\left(x_{0}\right), \gamma_{0}\right) .
\end{aligned}
$$

By the condition (iii), we have

$$
\left\langle Q\left(z_{0}\right), \Omega\left(y_{0}, \psi\left(x_{0}\right), \gamma_{0}\right)\right\rangle+\Theta\left(y_{0}, \psi\left(x_{0}\right), \gamma_{0}\right) \notin-\operatorname{int} C\left(x_{0}\right) .
$$


We see a contradiction between (3.1) and (3.3), and so we have $x_{0} \in \Psi\left(\gamma_{0}, \mu_{0}\right) \subseteq V$, which contradicts the fact $x_{\alpha} \notin V, \forall \alpha$. Hence, $\Psi(\cdot, \cdot)$ is upper semicontinuous in $\Lambda \times M$.

Now we prove that $\Psi\left(\gamma_{0}, \mu_{0}\right)$ is compact. We first show that $\Psi\left(\gamma_{0}, \mu_{0}\right)$ is a closed set. Indeed, we supposed that $\Psi\left(\gamma_{0}, \mu_{0}\right)$ is not a closed set, then there exists a net $\left\{x_{\alpha}\right\} \in \Psi\left(\gamma_{0}, \mu_{0}\right)$ such that $x_{\alpha} \rightarrow x_{0}$, but $x_{0} \notin \Psi\left(\gamma_{0}, \mu_{0}\right)$. The further argument is the same as above. And so we have $\Psi\left(\gamma_{0}, \mu_{0}\right)$ is a closed set. Moreover, as $\Psi\left(\gamma_{0}, \mu_{0}\right) \subseteq E\left(\gamma_{0}\right)$ and $E\left(\gamma_{0}\right)$ is compact, it follows that $\Psi\left(\gamma_{0}, \mu_{0}\right)$ is compact. Hence, by Lemma 2.2(iv), it follows that $\Psi(\cdot, \cdot)$ is closed in $\Lambda \times M$.

Remark 3.2 In the special case as that in Section 1 (d), Theorem 3.1 extends Theorem 3.1 of Chen et al. in [4].

Theorem 3.3 Assume for the problem (MQVIP) that

(i) $E(\cdot)$ is outer-continuous in $\Lambda$ and $K(\cdot, \cdot)$ is lower semicontinuous in $X \times \Lambda$;

(ii) $T(\cdot, \cdot)$ is upper semicontinuous with compact values in $X \times M$;

(iii) $W(\cdot)=Y \backslash-\operatorname{int} C(\cdot)$ is closed in $X$.

Then $\Psi(\cdot, \cdot)$ is outer-continuous in $\Lambda \times M$.

Proof Let $x_{0} \in \lim \sup _{\gamma \rightarrow \gamma_{0}, \mu \rightarrow \mu_{0}} \Psi(\gamma, \mu)$. There are nets $\left\{\left(\gamma_{\alpha}, \mu_{\alpha}\right)\right\}$ converging to $\left(\gamma_{0}, \mu_{0}\right)$ and $\left\{x_{\alpha}\right\}$ converging to $x_{0}$ with $x_{\alpha} \in \Psi\left(\gamma_{\alpha}, \mu_{\alpha}\right)$. By the outer continuity of $E(\cdot)$, we have $x \in E\left(\gamma_{0}\right)$. Now we show that $x_{0} \in \Psi\left(\gamma_{0}, \mu_{0}\right)$. Indeed, by the lower-semicontinuity of $K(\cdot, \cdot)$ in $X \times \Lambda$, for any $y_{0} \in K\left(x_{0}, \gamma_{0}\right)$, there exists $y_{\alpha} \in K\left(x_{\alpha}, \gamma_{\alpha}\right)$ such that $y_{\alpha} \rightarrow y_{0}$. As $x_{\alpha} \in$ $\Psi\left(\gamma_{\alpha}, \mu_{\alpha}\right)$, there exists $z_{\alpha} \in T\left(x_{\alpha}, \mu_{\alpha}\right)$ such that

$$
\left\langle Q\left(z_{\alpha}\right), \Omega\left(y_{\alpha}, \psi\left(x_{\alpha}\right), \gamma_{\alpha}\right)\right\rangle+\Theta\left(y_{\alpha}, \psi\left(x_{\alpha}\right), \gamma_{\alpha}\right) \notin-\operatorname{int} C\left(x_{\alpha}\right) .
$$

Since $T(\cdot, \cdot)$ is upper semicontinuous with compact-values in $X \times M$, there exists $z_{0} \in$ $T\left(x_{0}, \mu_{0}\right)$ such that $z_{\alpha} \rightarrow z_{0}$ (can take a subnet if necessary). Since $Q(\cdot), \Omega(\cdot, \psi(\cdot), \cdot)$ are continuous, we have

$$
\left\langle Q\left(z_{\alpha}\right), \Omega\left(y_{\alpha}, \psi\left(x_{\alpha}\right), \gamma_{\alpha}\right)\right\rangle \rightarrow\left\langle Q\left(z_{0}\right), \Omega\left(y_{0}, \psi\left(x_{0}\right), \gamma_{0}\right)\right\rangle .
$$

It follows from the continuity of $\Theta(\cdot, \psi(\cdot), \cdot)$ that

$$
\begin{aligned}
& \left\langle Q\left(z_{\alpha}\right), \Omega\left(y_{\alpha}, \psi\left(x_{\alpha}\right), \gamma_{\alpha}\right)\right\rangle+\Theta\left(y_{\alpha}, \psi\left(x_{\alpha}\right), \gamma_{\alpha}\right) \\
& \quad \rightarrow\left\langle Q\left(z_{0}\right), \Omega\left(y_{0}, \psi\left(x_{0}\right), \gamma_{0}\right)\right\rangle+\Theta\left(y_{0}, \psi\left(x_{0}\right), \gamma_{0}\right) .
\end{aligned}
$$

By the condition (iii) and (3.4), we have

$$
\left\langle Q\left(z_{0}\right), \Omega\left(y_{0}, \psi\left(x_{0}\right), \gamma_{0}\right)\right\rangle+\Theta\left(y_{0}, \psi\left(x_{0}\right), \gamma_{0}\right) \notin-\operatorname{int} C\left(x_{0}\right) .
$$

Hence, $x_{0} \in \Psi\left(\gamma_{0}, \mu_{0}\right)$. Thus, $\Psi(\cdot, \cdot)$ is outer-continuous in $\Lambda \times M$.

\section{Theorem 3.4 Assume for the problem (MQVIP) that}

(i) $E(\cdot)$ is outer-open in $\Lambda$ and $K\left(x_{0}, \cdot\right)$ is lower semicontinuous in $\Lambda$ for all $x_{0} \in E\left(\gamma_{0}\right)$;

(ii) for all $x_{0} \in E\left(\gamma_{0}\right), T\left(x_{0}, \cdot\right)$ is upper semicontinuous with compact values in $M$; 
(iii) for all $x_{0} \in E\left(\gamma_{0}\right), W\left(x_{0}\right)=Y \backslash-$ int $C\left(x_{0}\right)$ is closed.

Then $\Psi(\cdot, \cdot)$ is outer-open in $\Lambda \times M$.

Proof Let $x_{0} \in \operatorname{limsupo}_{\gamma \rightarrow \gamma_{0}, \mu \rightarrow \mu_{0}} \Psi(\gamma, \mu)$. There are a neighborhood $V$ of $x_{0}$ and nets $\left\{\gamma_{\alpha}\right\} \subseteq \Lambda, \gamma_{\alpha} \neq \gamma_{0}$ converging to $\gamma_{0}$ and $\left\{\mu_{\alpha}\right\} \subseteq M, \mu_{\alpha} \neq \mu_{0}$ converging to $\mu_{0}$ such that $V \subset \Psi\left(\gamma_{\alpha}, \mu_{\alpha}\right), \forall \alpha$. By $V \subset E\left(\gamma_{\alpha}\right)$, we have $x \in \operatorname{limsupo}_{\gamma \rightarrow \gamma_{0}} E(\gamma)$. It follows from (i) that $x_{0} \in E\left(\gamma_{0}\right)$. Now we show that $x_{0} \in \Psi\left(\gamma_{0}, \mu_{0}\right)$. Indeed, by the lower-semicontinuity of $K\left(x_{0}, \cdot\right)$ in $\Lambda$, for any $y_{0} \in K\left(x_{0}, \gamma_{0}\right)$, there exists $y_{\alpha} \in K\left(x_{0}, \gamma_{\alpha}\right)$ such that $y_{\alpha} \rightarrow y_{0}$. As $x_{0} \in \Psi\left(\gamma_{\alpha}, \mu_{\alpha}\right)$, there exists $z_{\alpha} \in T\left(x_{0}, \mu_{\alpha}\right)$ such that

$$
\left\langle Q\left(z_{\alpha}\right), \Omega\left(y_{\alpha}, \psi\left(x_{0}\right), \gamma_{\alpha}\right)\right\rangle+\Theta\left(y_{\alpha}, \psi\left(x_{0}\right), \gamma_{\alpha}\right) \notin-\operatorname{int} C\left(x_{0}\right) \text {. }
$$

Since $T\left(x_{0}, \cdot\right)$ is upper semicontinuous with compact-values in $M$, there exists $z_{0} \in$ $T\left(x_{0}, \mu_{0}\right)$ such that $z_{\alpha} \rightarrow z_{0}$ (can take a subnet if necessary). Since $Q(\cdot), \Omega(\cdot, \psi(\cdot), \cdot)$ are continuous, we have

$$
\left\langle Q\left(z_{\alpha}\right), \Omega\left(y_{\alpha}, \psi\left(x_{0}\right), \gamma_{\alpha}\right)\right\rangle \rightarrow\left\langle Q\left(z_{0}\right), \Omega\left(y_{0}, \psi\left(x_{0}\right), \gamma_{0}\right)\right\rangle
$$

It follows from the continuity of $\Theta(\cdot, \psi(\cdot), \cdot)$ that

$$
\begin{aligned}
& \left\langle Q\left(z_{\alpha}\right), \Omega\left(y_{\alpha}, \psi\left(x_{0}\right), \gamma_{\alpha}\right)\right\rangle+\Theta\left(y_{\alpha}, \psi\left(x_{0}\right), \gamma_{\alpha}\right) \\
& \quad \rightarrow\left\langle Q\left(z_{0}\right), \Omega\left(y_{0}, \psi\left(x_{0}\right), \gamma_{0}\right)\right\rangle+\Theta\left(y_{0}, \psi\left(x_{0}\right), \gamma_{0}\right) .
\end{aligned}
$$

By the condition (iii) and (3.5), we have

$$
\left\langle Q\left(z_{0}\right), \Omega\left(y_{0}, \psi\left(x_{0}\right), \gamma_{0}\right)\right\rangle+\Theta\left(y_{0}, \psi\left(x_{0}\right), \gamma_{0}\right) \notin-\operatorname{int} C\left(x_{0}\right) .
$$

Hence, $x_{0} \in \Psi\left(\gamma_{0}, \mu_{0}\right)$. Thus, $\Psi(\cdot, \cdot)$ is outer-open in $\Lambda \times M$.

The following example shows that all assumptions of Theorem 3.4 are fulfilled. But the outer-continuity in Theorem 3.3 is not satisfied. Thus, Theorem 3.3 cannot be applied.

Example 3.5 Let $X=Y=\mathbb{R}, \Lambda=M=[0,1], \gamma_{0}=0, C=[0,+\infty)$, let $\psi, Q$ be identity mappings, and $T(x, \gamma)=\left[0,2^{x^{2}+y^{2}+2 \gamma+2}\right], K(x, \gamma)=(-1, \gamma)$ and $\Omega(y, x, \gamma)=0$ and

$$
\Theta(y, x, \gamma)= \begin{cases}0 & \text { if } \gamma=0, \\ {\left[\frac{1}{2^{\gamma+1}}, 1\right]} & \text { otherwise }\end{cases}
$$

We have $E(\gamma)=(-1, \gamma), \forall \gamma \in[0,1]$. We show that the conditions (i), (ii) and (iii) of Theorem 3.4 are easily seen to be fulfilled. And so $\Psi(\cdot, \cdot)$ is outer-open at $(0,0)$ (in fact, $\Psi(0,0)=(-1,0)$ and $\Psi(\gamma, \mu)=(-1, \gamma)$ for all $\gamma \in(0,1])$, but $E(\cdot)$ is not outer-continuous at 0 . Hence $\Psi(\cdot, \cdot)$ is not outer-continuous at $(0,0)$.

The following example shows that all assumptions of Theorem 3.4 and Theorem 3.3 are fulfilled. But Theorem 3.1 cannot be applied. 
Example 3.6 Let $Q, X, Y, \Lambda, M, T, C, \psi, \Omega, \gamma_{0}$ as in Example 3.5, and let $K(x, \gamma)=$ $\{(\zeta, \gamma \zeta): \zeta \in \mathbb{R}\}$ and

$$
\Theta(y, x, \gamma)= \begin{cases}0 & \text { if } \lambda=0, \\ {\left[\frac{1}{e^{\cos ^{2} \lambda+2}}, 1\right]} & \text { otherwise }\end{cases}
$$

Then, we have $E(\gamma)=\{(\zeta, \gamma \zeta): \zeta \in \mathbb{R}\}$ for all $\gamma \in[0,1]$. Hence, $E$ is outer-open and outercontinuous at 0 . It is not hard to see that (i)-(iii) in Theorem 3.4 and Theorem 3.3 are satisfied. Hence, $\Psi(\cdot, \cdot)$ is outer-open and outer-continuous at $(0,0)$ (in fact, $\Psi(\gamma, \mu)=$ $\{(\zeta, \gamma \zeta): \zeta \in \mathbb{R}\}$ for all $\gamma \in[0,1])$. We see that $E(\cdot)$ is not upper semicontinuous at 0 . Thus, $\Psi(\cdot, \cdot)$ is not upper semicontinuous at $(0,0)$. Hence, we cannot apply Theorem 3.1.

The following example shows that the assumptions in Theorem 3.1, Theorem 3.3 and Theorem 3.4 may be satisfied in every case.

Example 3.7 Let $X, Y, \Lambda, M, \psi, Q, C, \gamma_{0}$ be as in Example 3.6, and let $T(x, \gamma)=\left\{\frac{1}{e}\right\}$, $\Omega(y, x, \gamma)=2^{\gamma^{2}+\sin ^{4} x+1}, K(x, \gamma)=[0,1]$ and

$$
\Theta(y, x, \gamma)= \begin{cases}0 & \text { if } \gamma=0, \\ {\left[\frac{1}{2}, 1\right]} & \text { otherwise. }\end{cases}
$$

We see that the conditions (i), (ii) and (iii) in Theorem 3.1, Theorem 3.3 and Theorem 3.4 are satisfied. And so, $\Psi(\cdot, \cdot)$ is outer-open, outer-continuous and upper semicontinuous at $(0,0)$ (in fact, $\Psi(\gamma, \mu)=[0,1], \forall \gamma \in[0,1]$ ).

\section{Lower semicontinuity of a solution set}

In this section, we establish that the condition $\left(H_{h}\left(\gamma_{0}, \mu_{0}\right)\right)$ is a sufficient and necessary condition for the lower semicontinuity, the Hausdorff lower semicontinuity, the continuity and Hausdorff continuity of the solution set for the parametric generalized vector mixed quasivariational inequality problem (MQVIP).

Motivated by the hypothesis $\left(H_{1}\right)$ of $[15,17]$ and the assumption $\left(H_{g}\right)$ in $[4,7]$, by virtue of the parametric gap function $h(\cdot, \cdot, \cdot)$, now we introduce the following key assumption.

$\left(H_{h}\left(\gamma_{0}, \mu_{0}\right)\right)$ Given $\left(\gamma_{0}, \mu_{0}\right) \in \Lambda \times M$. For any open neighborhood $N$ of the origin in $X$, there exist $\alpha>0$ and a neighborhood $V\left(\gamma_{0}, \mu_{0}\right)$ of $\left(\gamma_{0}, \mu_{0}\right)$ such that for all $(\gamma, \mu) \in$ $V\left(\gamma_{0}, \mu_{0}\right)$ and $x \in E(\gamma) \backslash(\Psi(\gamma, \mu)+N)$, one has $h(x, \gamma, \mu) \geq \alpha$.

As mentioned in Zhao [17] and Kien [15], the above hypothesis $\left(H_{h}\left(\gamma_{0}, \mu_{0}\right)\right)$ is characterized by a common theme used in mathematical analysis. Such a theme interprets a proposition associated with a set in terms of other propositions associated with the complement set. Instead of imposing restrictions on the solution set, the hypothesis $\left(H_{h}\left(\gamma_{0}, \mu_{0}\right)\right)$ lays a condition on the behavior of the parametric gap function on the complement of the solution set.

Geometrically, the hypothesis $\left(H_{h}\left(\gamma_{0}, \mu_{0}\right)\right)$ means that, given a small open neighborhood $N$ of the origin in $X$, we can find a small positive number $\alpha>0$ and a neighborhood $V\left(\gamma_{0}, \mu_{0}\right)$ of $\left(\gamma_{0}, \mu_{0}\right)$, such that for all $(\gamma, \mu)$ in the neighborhood of $\left(\gamma_{0}, \mu_{0}\right)$, if a feasible point $x$ is not in the set $\Psi(\gamma, \mu)+N$, then a 'gap' by an amount of at least $\alpha$ will be yielded. 
The following Lemma 4.1 is modified from Proposition 3.1 in Kien [15].

Lemma 4.1 Suppose that all conditions in Lemma 2.7 are satisfied. For any open neighborhood $N$ of the origin in $X$, let

$$
\Phi(\gamma, \mu):=\inf _{x \in E(\gamma) \backslash(\Psi(\gamma, \mu)+N)} h(x, \gamma, \mu) .
$$

Then $\left(H_{h}\left(\gamma_{0}, \mu_{0}\right)\right)$ holds if and only if for any open neighborhood $N$ of the origin in $X$, one has

$$
\lim _{\gamma \rightarrow \gamma_{0}, \mu \rightarrow \mu_{0}} \inf \Phi(\gamma, \mu)>0
$$

Proof If $\left(H_{h}\left(\gamma_{0}, \mu_{0}\right)\right)$ holds, then for any open neighborhood $N$ of the origin in $X$, there exist $\alpha>0$ and a neighborhood $V\left(\gamma_{0}, \mu_{0}\right)$ of $\left(\gamma_{0}, \mu_{0}\right)$ such that for all $(\gamma, \mu) \in V\left(\gamma_{0}, \mu_{0}\right)$ and $x \in E(\gamma) \backslash(\Psi(\gamma, \mu)+N)$, one has $h(x, \gamma, \mu) \geq \alpha$.

This implies that $\Phi(\gamma, \mu) \geq \alpha$ for every $(\gamma, \mu) \in V\left(\gamma_{0}, \mu_{0}\right)$, hence

$$
\lim _{\gamma \rightarrow \gamma_{0}, \mu \rightarrow \mu_{0}} \inf \Phi(\gamma, \mu) \geq \alpha>0
$$

Conversely, for any open neighborhood $N$ of the origin in $X$,

$$
\pi=\lim _{\gamma \rightarrow \gamma_{0}, \mu \rightarrow \mu_{0}} \inf \Phi(\gamma, \mu)>0
$$

then there exists a neighborhood $V\left(\gamma_{0}, \mu_{0}\right)$ of $\left(\gamma_{0}, \mu_{0}\right)$ such that

$$
\Phi(\gamma, \mu)=\inf _{x \in E(\gamma) \backslash(\Psi(\gamma, \mu)+N)} h(x, \gamma, \mu) \geq \alpha>0
$$

for all $(\gamma, \mu) \in V\left(\gamma_{0}, \mu_{0}\right)$, where $\alpha:=\frac{1}{2} \pi$. Hence, for any $x \in E(\gamma) \backslash(\Psi(\gamma, \mu)+N)$, we have

$$
h(x, \gamma, \mu) \geq \alpha>0,
$$

which shows that $\left(H_{h}\left(\gamma_{0}, \mu_{0}\right)\right)$ holds.

\section{Remark 4.2 ([21])}

(i) Let a set $A \subset X, A$ is said to be balanced if $\lambda A \subset A$ for every $\lambda \in R$ with $|\lambda| \leq 1$;

(ii) For each neighborhood $N$ of the origin in $X$, there exists a balanced open neighborhood $U$ of the origin in $X$ such that $U+U+U \subset N$.

Theorem 4.3 Suppose that the condition $\left(H_{h}\left(\gamma_{0}, \mu_{0}\right)\right)$ holds and

(i) $E(\cdot)$ is lower semicontinuous with compact values in $\Lambda$;

(ii) $K(\cdot, \cdot)$ is continuous with compact values in $X \times \Lambda$;

(iii) $T(\cdot, \cdot)$ is continuous with compact values in $X \times M$;

(iv) $C(\cdot)$ is upper semicontinuous in $X$ and $e(\cdot) \in \operatorname{int} C(\cdot)$ is continuous in $X$;

(v) $W(\cdot)=Y \backslash-\operatorname{int} C(\cdot)$ is closed in $X$.

Then $\Psi(\cdot, \cdot)$ is Hausdorfflower semicontinuous in $\Lambda \times M$. 
Proof Suppose to the contrary that $\left(H_{h}\left(\gamma_{0}, \mu_{0}\right)\right)$ holds but $\Psi(\cdot, \cdot)$ is not Hausdorff lower semicontinuous at $\left(\gamma_{0}, \mu_{0}\right) \in \Lambda \times M$. Then there exist a neighborhood $N$ of the origin in $X$, a net $\left\{\left(\gamma_{\alpha}, \mu_{\alpha}\right)\right\} \subset \Lambda \times M$ with $\left(\gamma_{\alpha}, \mu_{\alpha}\right) \rightarrow\left(\gamma_{0}, \mu_{0}\right)$ and a net $\left\{x_{\alpha}\right\}$ such that

$$
x_{\alpha} \in \Psi\left(\gamma_{0}, \mu_{0}\right) \backslash\left(\Psi\left(\gamma_{\alpha}, \mu_{\alpha}\right)+N\right) .
$$

By the compactness of $\Psi\left(\gamma_{0}, \mu_{0}\right)$, we can assume that $x_{\alpha} \rightarrow x_{0} \in \Psi\left(\gamma_{0}, \mu_{0}\right)$. By Lemma 4.2, there exists a balanced open neighborhood $U_{0}$ of the origin in $X$ such that $U_{0}+U_{0}+$ $U_{0} \subset N$. Hence, for any given $\varepsilon>0,\left(x_{0}+\varepsilon U_{0}\right) \cap E\left(\gamma_{0}\right) \neq \emptyset$. By $E(\cdot)$ is lower semicontinuous at $\gamma_{0} \in \Lambda$, there exists some $k_{1}$ such that $\left(x_{0}+\varepsilon U_{0}\right) \cap E\left(\gamma_{k}\right) \neq \emptyset$ for all $k \geq k_{1}$.

For $\varepsilon \in(0,1]$, suppose that $a_{k} \in\left(x_{0}+\varepsilon U_{0}\right) \cap E\left(\gamma_{k}\right)$. We claim that $a_{k} \notin \Psi\left(\gamma_{k}\right)+U_{0}$. Otherwise, there exists $t_{k} \in \Psi\left(\gamma_{k}\right)$ such that $a_{k}-t_{k} \in U_{0}$. Without loss of generality, we may assume that $x_{k}-x_{0} \in U_{0}$ whenever $k$ is sufficiently large. Consequently, we get

$$
x_{k}-t_{k}=\left(x_{k}-x_{0}\right)+\left(x_{0}-a_{k}\right)+\left(a_{k}-t_{k}\right) \in U_{0}+\left(-\varepsilon U_{0}\right)+U_{0} \subset U_{0}+U_{0}+U_{0} \subset N .
$$

This implies that $x_{k} \in \Psi\left(\gamma_{k}, \mu_{k}\right)+N$, contrary to (4.1). Thus,

$$
a_{k} \notin \Psi\left(\gamma_{k}, \mu_{k}\right)+U_{0}
$$

By the assumption $\left(H_{h}\left(\gamma_{0}, \mu_{0}\right)\right)$, there exists $\sigma>0$ such that $h\left(a_{k}, \gamma_{k}, \mu_{k}\right) \geq \sigma$. By Lemma 2.7, $h(\cdot, \cdot, \cdot)$ is upper semicontinuous in $X \times \Lambda \times M$. So, for any $\delta>0$ and for $k$ sufficiently large, we have

$$
h\left(a_{k}, \gamma_{k}, \mu_{k}\right)-\delta \leq h\left(x_{0}, \gamma_{0}, \mu_{0}\right) .
$$

We can take $\delta$ such that $\sigma-\delta>0$. Thus,

$$
h\left(x_{0}, \gamma_{0}, \mu_{0}\right) \geq h\left(a_{k}, \gamma_{k}, \mu_{k}\right)-\delta \geq \sigma-\delta>0 .
$$

Hence

$$
h\left(x_{0}, \gamma_{0}, \mu_{0}\right)=\min _{z \in T\left(x_{0}, \mu_{0}\right)} \max _{y \in K\left(x_{0}, \gamma_{0}\right)}\left\{-\xi_{e}\left(x_{0},\left\langle Q(z), \Omega\left(y, \psi\left(x_{0}\right), \gamma_{0}\right)\right\rangle+\Theta\left(y, \psi\left(x_{0}\right), \gamma_{0}\right)\right)\right\}>0,
$$

and so

$$
\max _{y \in K\left(x_{0}, \gamma_{0}\right)}\left\{-\xi_{e}\left(x_{0},\left\langle Q(z), \Omega\left(y, \psi\left(x_{0}\right), \gamma_{0}\right)\right\rangle+\Theta\left(y, \psi\left(x_{0}\right), \gamma_{0}\right)\right)\right\}>0, \quad \forall z \in T\left(x_{0}, \mu_{0}\right) .
$$

Since $y \in K\left(x_{0}, \lambda_{0}\right)$ is arbitrary, we have

$$
-\xi_{e}\left(x_{0},\left\langle Q(z), \Omega\left(y, \psi\left(x_{0}\right), \gamma_{0}\right)\right\rangle+\Theta\left(y, \psi\left(x_{0}\right), \gamma_{0}\right)\right)>0
$$

or

$$
\xi_{e}\left(x_{0},\left\langle Q(z), \Omega\left(y, \psi\left(x_{0}\right), \gamma_{0}\right)\right\rangle+\Theta\left(y, \psi\left(x_{0}\right), \gamma_{0}\right)\right)<0
$$


By Lemma 2.3(i), we have

$$
\left.\left\langle Q(z), \Omega\left(y, \psi\left(x_{0}\right), \gamma_{0}\right)\right\rangle+\Theta\left(y, \psi\left(x_{0}\right), \gamma_{0}\right)\right) \in-\operatorname{int} C\left(x_{0}\right),
$$

which contradicts $x_{0} \in \Psi\left(\gamma_{0}, \mu_{0}\right)$. Therefore, $\Psi(\cdot, \cdot)$ is Hausdorff lower semicontinuous in $\Lambda \times M$.

Corollary 4.4 Suppose that all conditions in Theorem 4.3 are satisfied. Then we have $\Psi(\cdot, \cdot)$ is lower semicontinuous in $\Lambda \times M$.

\section{Theorem 4.5 Suppose that}

(i) $E(\cdot)$ is continuous with compact values in $\Lambda$;

(ii) $K(\cdot, \cdot)$ is continuous with compact values in $X \times \Lambda$;

(iii) $T(\cdot, \cdot)$ is continuous with compact values in $X \times M$;

(iv) $C(\cdot)$ is upper semicontinuous in $X$ and $e(\cdot) \in \operatorname{int} C(\cdot)$ is continuous in $X$;

(v) $W(\cdot)=Y \backslash-\operatorname{int} C(\cdot)$ is closed in $X$.

Then $\Psi(\cdot, \cdot)$ is Hausdorfflower semicontinuous in $\Lambda \times M$ if and only if $\left(H_{h}\left(\gamma_{0}, \mu_{0}\right)\right)$ holds.

Proof From Theorem 4.3, we only need to prove the necessity. Suppose to the contrary that $\Psi(\cdot, \cdot)$ is Hausdorff lower semicontinuous at $\left(\gamma_{0}, \mu_{0}\right) \in \Lambda \times M$, but $\left(H_{h}\left(\gamma_{0}, \mu_{0}\right)\right)$ does not hold. By Lemma 4.1, there exists a neighborhood $N$ of the origin in $X$ such that

$$
\lim _{\gamma \rightarrow \gamma_{0}, \mu \rightarrow \mu_{0}} \inf \Phi(\gamma, \mu)=0
$$

Then there exists a net $\left\{\left(\gamma_{\alpha}, \mu_{\alpha}\right)\right\} \subset \Lambda \times M$ with $\left(\gamma_{\alpha}, \mu_{\alpha}\right) \rightarrow\left(\gamma_{0}, \mu_{0}\right)$ such that

$$
\lim _{\alpha \rightarrow \infty} \Phi\left(\gamma_{\alpha}, \mu_{\alpha}\right)=\lim _{\alpha \rightarrow \infty} \inf _{x \in E\left(\gamma_{\alpha}\right) \backslash\left(\Psi\left(\lambda_{\alpha}, \mu_{\alpha}\right)+N\right)} h\left(x, \gamma_{\alpha}, \mu_{\alpha}\right)=0
$$

By $E\left(\gamma_{\alpha}\right) \backslash\left(\Psi\left(\gamma_{\alpha}, \mu_{\alpha}\right)+N\right)$ is a compact set and $h(\cdot, \cdot, \cdot)$ is continuous from Lemma 2.7, there exists $x_{\alpha} \in E\left(\gamma_{\alpha}\right) \backslash\left(\Psi\left(\gamma_{\alpha}, \mu_{\alpha}\right)+N\right)$ satisfying $\Phi\left(\gamma_{\alpha}, \mu_{\alpha}\right)=h\left(x_{\alpha}, \gamma_{\alpha}, \mu_{\alpha}\right)$. Clearly, (4.2) implies

$$
\lim _{\alpha \rightarrow \infty} h\left(x_{\alpha}, \gamma_{\alpha}, \mu_{\alpha}\right)=0
$$

Since $E(\cdot)$ is upper semicontinuous with compact values in $\Lambda$, we can assume that $x_{\alpha} \rightarrow x_{0}$ with $x_{0} \in E\left(\gamma_{0}\right)$. By the continuity of $h(\cdot, \cdot, \cdot)$, we have $h\left(x_{0}, \gamma_{0}, \mu_{0}\right)=0$ and so $x_{0} \in \Psi\left(\gamma_{0}, \mu_{0}\right)$. For any $t \in \Psi\left(\gamma_{0}, \mu_{0}\right)$, since $\Psi(\cdot, \cdot)$ is Hausdorff lower semicontinuous at $\left(\gamma_{0}, \mu_{0}\right) \in \Lambda \times M$, we can find a net $\left\{t_{\alpha}\right\} \subset \Psi\left(\gamma_{\alpha}, \mu_{\alpha}\right)$ such that $t_{\alpha} \rightarrow t_{0}, \forall \alpha$. By $x_{\alpha} \in E\left(\gamma_{\alpha}\right) \backslash\left(\Psi\left(\gamma_{\alpha}, \mu_{\alpha}\right)+N\right)$, $t_{\alpha}-x_{\alpha} \nsubseteq N$. Letting $\alpha \rightarrow \infty$, we have $t_{0}-x_{0} \nsubseteq N, \forall t_{0} \in \Psi\left(\gamma_{0}, \mu_{0}\right)$. Since $x_{0} \in \Psi\left(\gamma_{0}, \mu_{0}\right)$, we have a contradiction. Thus, $\left(H_{h}\left(\gamma_{0}, \mu_{0}\right)\right)$ holds.

The following example shows that $\left(H_{h}\left(\gamma_{0}, \mu_{0}\right)\right)$ in Theorem 4.5 is essential.

Example 4.6 Let $X, \Lambda, M, \gamma_{0}, \psi, Q$ as in Example 3.5, let $Y=\mathbb{R}^{2}, C=\mathbb{R}_{+}^{2}, K(x, \gamma)=[-1,1]$, $T(x, \mu)=\left[1, \gamma+x^{2}\right], \Theta(y, \psi(x), \gamma)=0, \Omega(y, \psi(x), \gamma)=y-x$. Now we consider the problem (MQVIP) of finding $x \in E(\gamma)$ and $z \in T(x, \mu)$ such that

$$
\langle Q(z), \Omega(y, \eta(x), \gamma)\rangle+\Theta(y, \eta(x), \gamma)=\left((y-x),\left(\gamma+x^{2}\right)(y-x)\right) \notin-\operatorname{int} \mathbb{R}_{+}^{2} .
$$


It follows from a direct computation

$$
\Psi(\gamma, \mu)= \begin{cases}\{-1,0\} & \text { if } \gamma=0 \\ \{-1\} & \text { otherwise }\end{cases}
$$

Hence $\Psi(\cdot, \cdot)$ is not H-lsc in $\Lambda \times M$. Now we show that condition $\left(H_{h}\left(\gamma_{0}, \mu_{0}\right)\right)$ does not hold at $(0,0)$. Taking $e=(1,1) \in \operatorname{int} \mathbb{R}_{+}^{2}$, we have

$$
\begin{aligned}
h(x, \gamma, \mu) & =\min _{z \in T(x, \gamma)} \max _{y \in K(x, \gamma)}\left\{-\xi_{e}(x,\langle Q(z), \Omega(y, \eta(x), \gamma)\rangle+\Theta(y, \eta(x), \gamma))\right\} \\
& =\max _{y \in K(x, \gamma)}\left(\left(\lambda+x^{2}\right)(x-y)\right) \\
& =\left(\gamma+x^{2}\right)(x+1) .
\end{aligned}
$$

We have $h(\cdot, \cdot, \cdot)$ is a parametric gap function of (MQVIP). For given $\left(\gamma_{0}, \mu_{0}\right) \in \Lambda \times M$, for any open neighborhood $N_{\varepsilon}(0)=(-\varepsilon, \varepsilon)$, choose $\varepsilon$ such that $0<\varepsilon<1$. For any $\alpha>0$, taking $\left(\gamma_{\beta}, \mu_{\beta}\right) \rightarrow(0,0)$ with $0<\gamma_{\beta}<\alpha$ and $x_{\beta}=0 \in E\left(\gamma_{\beta}\right) \backslash\left(\Psi\left(\gamma_{\beta}, \mu_{\beta}\right)+N_{\varepsilon}(0)\right)$, we have $h\left(x_{\beta}, \gamma_{\beta}, \mu_{\beta}\right)=\gamma_{\beta}<\alpha$. Hence, $\left(H_{h}\left(\gamma_{0}, \mu_{0}\right)\right)$ does not hold at $(0,0)$.

\section{Corollary 4.7}

(i) Suppose that all conditions in Theorem 4.5 are satisfied. Then we have $\Psi(\cdot, \cdot)$ is lower semicontinuous in $\Lambda \times M$ if and only if $\left(H_{h}\left(\gamma_{0}, \mu_{0}\right)\right)$ holds.

(ii) Suppose that all conditions in Theorem 4.5 are satisfied. Then we have $\Psi(\cdot, \cdot)$ is both continuous (H-continuous) and closed in $\Lambda \times M$ if and only if $\left(H_{h}\left(\gamma_{0}, \mu_{0}\right)\right)$ holds.

\section{Remark 4.8}

(i) In special cases as those in Section 1 (e) and (f), Theorem 4.5 extends Theorem 3.2 in [6] and Theorem 3.1 in [5]. Moreover, our assumption $\left(H_{h}\left(\gamma_{0}, \mu_{0}\right)\right)$ is different from the assumption $\left(H_{g}\right)$ in $[5,6]$. Besides, our problem (MQVIP) is considered in Hausdorff topological vector spaces.

(ii) In the special case as that in Section 1 (d), Theorem 4.5 extends Theorem 4.1 in [4], and in the special case as that in Section 1 (b), Corollary 4.7 (ii) extends Theorem 3.4 in [2]. Indeed, our assumption $\left(H_{h}\left(\gamma_{0}, \mu_{0}\right)\right)$ is a sufficient and necessary condition for the lower semicontinuity, the Hausdorff lower semicontinuity, the continuity and Hausdorff continuity of the solution set for (MQVIP) while the assumption $\left(H_{g}\right)$ in $[2,4]$ is only a sufficient condition.

\section{Competing interests}

The author declares that they have no competing interests.

\section{Authors' contributions}

The author is grateful to Professor Phan Quoc Khanh and Professor Lam Quoc Anh for their help in the research process. The author also thanks the two anonymous referees for their valuable remarks and suggestions, which helped to improve the article considerably. 


\section{References}

1. Ding, XP, Salahuddin: Generalized vector mixed general quasi-variational-like inequalities in Hausdorff topological vector spaces. Optim. Lett. (2012). doi:10.1007/s11590-012-0464-x

2. Agarwal, RP, Chen, JW, Cho, YJ, Wan, Z: Stability analysis for parametric generalized vector quasi-variational-like inequality problems. J. Inequal. Appl. (2012). doi:10.1186/1029-242X-2012-57

3. Li, J, He, ZQ: Gap functions and existence of solutions to generalized vector variational inequalities. Appl. Math. Lett. 18, 989-1000 (2005)

4. Chen, CR, Li, SJ, Fang, ZM: On the solution semicontinuity to a parametric generalized vector quasivariational inequality. Comput. Math. Appl. 60, 2417-2425 (2010)

5. Zhong, RY, Huang, NJ: Lower semicontinuity for parametric weak vector variational inequalities in reflexive Banach spaces. J. Optim. Theory Appl. 150, 2417-2425 (2011)

6. Zhong, RY, Huang, NJ: On the stability of solution mapping for parametric generalized vector quasiequilibrium problems. Comput. Math. Appl. 63, 807-815 (2012)

7. Li, SJ, Chen, CR: Stability of weak vector variational inequality problems. Nonlinear Anal. TMA 70, 1528-1535 (2009)

8. Anh, LQ, Khanh, PQ: Semicontinuity of the solution sets of parametric multivalued vector quasiequilibrium problems. J. Math. Anal. Appl. 294, 699-711 (2004)

9. Chen, CR, Li, SJ: Semicontinuity of the solution set map to a set-valued weak vector variational inequality. J. Ind. Manag. Optim. 3, 519-528 (2007)

10. Hung, NV: Continuity of solutions for parametric generalized quasi-variational relation problems. Fixed Point Theory Appl. 2012, Article ID 102 (2012)

11. Hung, NV: Sensitivity analysis for generalized quasi-variational relation problems in locally G-convex spaces. Fixed Point Theory Appl. 2012, Article ID 158 (2012)

12. Hung, NV: Existence conditions for symmetric generalized quasi-variational inclusion problems. J. Inequal. Appl. 2013, Article ID 40 (2013)

13. Khanh, PQ, Luu, LM: Upper semicontinuity of the solution set of parametric multivalued vector quasivariational inequalities and applications. J. Glob. Optim. 32, 551-568 (2005)

14. Khanh, PQ, Luu, LM: Lower and upper semicontinuity of the solution sets and approximate solution sets to parametric multivalued quasivariational inequalities. J. Optim. Theory Appl. 133, 329-339 (2007)

15. Kien, BT: On the lower semicontinuity of optimal solution sets. Optimization 54, 123-130 (2005)

16. Li, SJ, Chen, GY, Teo, KL: On the stability of generalized vector quasivariational inequality problems. J. Optim. Theory Appl. 113, 283-295 (2002)

17. Zhao, J: The lower semicontinuity of optimal solution sets. J. Math. Anal. Appl. 207, 240-254 (1997)

18. Khanh, PQ, Luc, DT: Stability of solutions in parametric variational relation problems, submitted for publication. Set-Valued Anal. 16, 1015-1035 (2008)

19. Rockafellar, RT, Wets, RJ-B: Variational Analysis. Springer, Berlin (1998)

20. Aubin, JP, Ekeland, I: Applied Nonlinear Analysis. Wiley, New York (1984)

21. Berge, C: Topological Spaces. Oliver \& Boyd, London (1963)

22. Chen, GY, Huang, XX, Yang, XQ: Vector Optimization: Set-Valued and Variational Analysis. Lecture Notes in Economics and Mathematical Systems, vol. 541. Springer, Berlin (2005)

23. Chen, $\mathrm{GY}$, Yang, $X Q, Y u, H$ : A nonlinear scalarization function and generalized quasi-vector equilibrium problems. J. Glob. Optim. 32, 451-466 (2005)

24. Mastroeni, G: Gap functions for equilibrium problems. J. Glob. Optim. 27, 411-426 (2003)

25. Li, SJ, Teo, KL, Yang, XQ, Wu, SY: Gap functions and existence of solutions to generalized vector quasi-equilibrium problems. J. Glob. Optim. 34, 427-440 (2006)

26. Li, J, Mastroeni, G: Vector variational inequalities involving set-valued mappings via scalarization with applications to error bounds for gap functions. J. Optim. Theory Appl. 145, 355-372 (2010)

27. Yang, XQ, Yao, JC: Gap functions and existence of solutions to set-valued vector variational inequalities. J. Optim. Theory Appl. 115, 407-417 (2002)

doi:10.1186/1029-242X-2013-276

Cite this article as: Hung: Stability of a solution set for parametric generalized vector mixed quasivariational inequality problem. Journal of Inequalities and Applications 2013 2013:276.

\section{Submit your manuscript to a SpringerOpen ${ }^{\circ}$ journal and benefit from:}

- Convenient online submission

Rigorous peer review

- Immediate publication on acceptance

- Open access: articles freely available online

- High visibility within the field

- Retaining the copyright to your article 